\title{
Acute encephalitis with refractory, repetitive partial seizures: A case report and literature review
}

\author{
BIN ZHANG $^{1}$ and LIN WANG ${ }^{2}$ \\ ${ }^{1}$ Emergency Intensive Care Unit, Qinghai Provincial People's Hospital, Xining, Qinghai 810007; ${ }^{2}$ Intensive Care Unit, \\ Department of Neurology, Xuanwu Hospital of Capital Medical University, Beijing 100053, P.R. China
}

Received April 5, 2015; Accepted May 12, 2016

DOI: $10.3892 /$ etm.2016.3750

\begin{abstract}
A male 46 year-old patient was admitted to the Emergency Intensive Care Unit of Qinghai Provincial People's Hospital (Xining, China) in December 2008 with complaints of a headache and fever that had lasted for three days. In addition, the patient had experienced two general convulsions accompanied with consciousness disorders for $7 \mathrm{~h}$. Although the patient's clinical manifestations, laboratory tests and cerebrospinal fluid examinations were consistent with numerous features associated with acute encephalitis, the patient's clinical course was different to that of acute encephalitis, and the prognosis was poor. The clinical course of the patient fully complied with the diagnostic criteria of acute encephalitis with refractory, repetitive partial seizures (AERRPS), as follows: i) The acute phase of encephalitis lasted >2 weeks; ii) partial seizures accompanied with general seizures occurred throughout the acute and convalescent course; iii) frequent seizures, a number of which resulted in a sustained state, were more prominent in the acute phase; iv) seizures were difficult to control; and v) known viral encephalitis and systemic metabolic disorders were excluded from the diagnosis. Based on previous reports and the present study, it can be concluded that AERRPS does not respond to the majority of anti-epileptic drugs, and while high-dose phenobarbital was effective in the current study, it was not able to fully control the focal and general seizures. Despite this, phenobarbital is required for maintenance in the recovery phase.
\end{abstract}

\section{Introduction}

Acute encephalitis with refractory, repetitive partial seizures (AERRPS) was first reported by the Japanese scholar Sakuma et al (1) in 2001. As the clinical manifestations, neuroimaging findings and prognosis of AERRPS are different

Correspondence to: Dr Bin Zhang, Emergency Intensive Care Unit, Qinghai Provincial People's Hospital, 2 Gonghe Road, Xining, Qinghai 810007, P.R. China

E-mail: binzhangdoc@126.com

Key words: encephalitis, status epilepticus, refractory epilepsy, phenobarbital from the known acute encephalitis, the international medical community classified AERRPS as a novel disease.

Primary clinical findings include the following: Acute onset of seizures or consciousness impairment; frequent and refractory partial seizures; the need for long-term anesthesia (more than 2 weeks) with intravenous barbiturates or benzodiazepines; a fever during the acute phase of illness; cerebrospinal fluid (CSF) analysis revealing mild pleocytosis or slight increase in protein; electroencephalogram (EEG) revealing slow background during the acute phase and multifocal spikes during the chronic phase; and magnetic resonance imaging (MRI) revealing no specific abnormalities aside from occasional T2/fluid attenuated inversion recovery hyperintense signals of the mesial temporal lobe (1-7). To date, $>200$ cases have been reported worldwide. AERRPS primarily affects school-age children and adolescents (8). The present study reports one case that was presented in 2008, and describes the treatment that was provided.

\section{Case report}

The present study reports the case of a male 46 year-old patient with no history of head trauma, seizures and other diseases, who smokes 20 cigarettes daily and has a long history of alcohol consumption. The patient suffered a persistent headache from December 14, 2008 after developing a cold. The headache reportedly increased during exercise, and was accompanied with general aches, fever, and a fluctuating body temperature between 38.5 and $39^{\circ} \mathrm{C}$. At 7:00 am, December 17th during exercise at home, the patient suddenly exhibited general convulsions, unconsciousness, showed the whites of his eyes, and experienced trismus, facial cyanosis, and limb stiffness followed by clonus, which lasted $\sim 2 \mathrm{~min}$ before the convulsions remitted. The patient awoke a number of minutes later. The patient suffered from the above convulsions at 8:00 a.m., December 17th in the emergency room of Qinghai Provincial People's Hospital (Xingin, China), and was fractionally intravenously injected $50 \mathrm{mg}$ diazepam (Xudong Haipu Pharmaceutical, Ltd., Shanghai, China). The convulsions arrested $\sim 5$ min following the injection; however, the patient still exhibited dysphoria, despite the cranial computed tomography (CT) examination detecting no abnormalities. The patient was, therefore, admitted into the Emergency Intensive Care Unit (EICU) at Qinghai Provincial People's Hospital on December 17th. The present study was 
conducted in accordance with the declaration of Helsinki and was performed with approval from the Ethics Committee of Qinghai Provincial People's Hospital. Written informed consent was obtained from the patient's family.

Examinations. On December 17th, the patient's body temperature was $38.4^{\circ} \mathrm{C}$, pulse was 90 beats $/ \mathrm{min}$, breathing was $29 \mathrm{times} / \mathrm{min}$ and blood pressure was $121 / 77 \mathrm{mmHg}$. The patient was in a light coma. The bilateral pupils exhibited the same size and roundness $(3.0 \mathrm{~mm})$, and were sensitive to light. The cardiopulmonary abdominal examination was normal. Neurological examination revealed cervical resistance and a jaw-chest distance of three cross-fingers, and no other positive signs were found in the nervous system.

Auxiliary examinations. On December 17th, the patient's cranial CT and chest X-ray showed no abnormalities. White blood cell count was $12.46 \times 10^{9}$ cells/1 (measured using an Automatic Hematology Analyzer; cat. no. XT-1800i; Sysmex Corporation, Kobe, Japan). A lumbar puncture was performed three times on the first, fifth and sixteenth day, and the CSF pressures were $190 \mathrm{~cm} \mathrm{H}_{2} \mathrm{O}, 215 \mathrm{~cm} \mathrm{H}_{2} \mathrm{O}$ and $175 \mathrm{~cm} \mathrm{H}_{2} \mathrm{O}$, respectively; the cell counting (white blood cells in CSF) was 13 cells $/ \mathrm{mm}$, 8 cells $/ \mathrm{mm}$ and 5 cells $/ \mathrm{mm}$, respectively; protein concentration was $1.22 \mathrm{~g} / 1,1.14 \mathrm{~g} / 1$ and $0.37 \mathrm{~g} / 1$, respectively (cell counting and protein concentration measured using Cobas 8000 Modular Analyzer; Roche Diagnostics, Basel, Switzerland); and the detection of cryptococcus was negative (identified using an India Ink Staining kit; cat. no. BA4042; Beisuo Biological Technology, Ltd., Zhuhai, China). Viral immunological analysis of blood and CSF showed no abnormalities.

Clinical course. The patient was diagnosed with viral encephalitis, and measurements of intracranial pressure reduction were performed, and ribavirin and phenobarbital was administered. Every 8 h, 0.1 mg phenobarbital (New Asia Pharmaceutical, Ltd., Shanghai, China) was intravenously injected. Every 12 h, 0.5 g ribavirin was intravenously injected (Romit Pharmaceutical Corporation, Xinghua, China) The patient exhibited convulsions again on the second day, which were the same as those experienced prior to hospitalization; the convulsions lasted $\sim 3$ min and were accompanied with apnea, therefore, the patient underwent trans-oral intubation for mechanical ventilation and was administered an intravenous injection of $800 \mathrm{mg}$ sodium valproate (Nankai Yongong Pharmaceutical, Ltd., Tongliao, China), which was then consistently administered to the patient ( $1 \mathrm{mg} / \mathrm{min} / \mathrm{kg}$ ). However, the patient continued to experience frequent convulsions, $>10$ times daily, which alternated between general tonic-clonic seizures and focal facial-finger twitching seizures. Combinations of anti-epileptic drugs and powerful sedatives had no significant clinical effects (sodium valproate $1 \mathrm{mg} / \mathrm{min} / \mathrm{kg}$ via pump; phenobarbital $0.1 \mathrm{mg}$ intravenously injected every 8 h; midazolam $32 \mathrm{mg} / \mathrm{h}$ (Corden Pharma S.P.A., Monza, Italy) via pump; propofol $320 \mathrm{mg} / \mathrm{h}$ (Jiangsu Nhwa Pharmaceutical Co., Ltd., Xuzhou, China) via pump; phenytoin 0.2 g (Zhejiang Million State Pharmaceutical, Ltd., Wenling, China) via nasogastric intubation every $8 \mathrm{~h}$; and lamotrigine 25 mg (GlaxoSmithKline, Poznań, Poland) via nasogastric intubation every $12 \mathrm{~h}$ ). The plasma concentrations of valproate, phenobarbital and phenytoin were monitored and maintained within the effective therapeutic concentration ranges. As a result of the poor effects of the above drugs, the patient was administered the muscle relaxant vecuronium (Zhejiang Xianju Pharmaceutical, Ltd., Hangzhou, China) after one week, intravenously infused $2-4 \mathrm{mg} / \mathrm{h}$. Although the systemic and local twitching was markedly reduced, EEG monitoring demonstrated the persistent status of a large quantity of high-amplitude sharp waves and spikes at all leads (Fig. 1).

After 5 days of the application of muscle relaxants, the medication was withdrawn due to the absence of any effect. On day 14 of hospitalization, the patient was diagnosed with AERRPS. The administration of anti-epileptic drugs was arrested, and the patient was administered an intravenous injection of $0.2 \mathrm{~g}$ phenobarbital once every $2 \mathrm{~h}$; the total daily quantity was $2.4 \mathrm{~g}$, and the plasma concentration was between 15 and $198 \mu \mathrm{g} / \mathrm{ml}$. When the plasma concentration was $<100 \mu \mathrm{g} / \mathrm{ml}$, no noticeable reduction of seizures was observed. On day 19, the plasma concentration reached $137 \mu \mathrm{g} / \mathrm{ml}$ and general seizures were markedly reduced, although the partial seizures remained frequent. On day 23 , the plasma concentration reached $198 \mu \mathrm{g} / \mathrm{ml}$, and the general and partial seizures were markedly decreased to 0-2 times daily (general seizures) and 5-10 times daily (partial seizures). The dose of phenobarbital was then gradually decreased, and when a large dose of phenobarbital was administered on day 64 ( 0.2 g every 6 h), no general convulsive seizures occurred. However, partial seizures still occurred occasionally, therefore, the therapy was changed to oral administration of $0.18 \mathrm{~g}$ phenobarbital three times per day. Clinical characteristics of the patient included a long-term lasting fever and a daily temperature fluctuating between 38.0 and $39.5^{\circ} \mathrm{C}$. After day 29 when the convulsions became gradually controlled, the body temperature gradually reduced to the normal range. The patient became conscious on day 55, and although language function, cognitive ability, capacity of calculation and memory were impaired, the patient could walk under assistance. The patient's intelligence was determined to be equivalent to that of 2-3 year-old child. Cranial MRI on day 56 and 217 after admission showed no obvious abnormalities. The patient was transferred from EICU to the Department of Neurology Rehabilitation of Qinghai Provincial People's Hospital 93 days after admission, but succumbed to suffocation on day 237.

\section{Discussion}

In 1986, Awaya and Fukuyama (2) first described a group of Japanese children who suffered from acute encephalitis, with uncontrollable partial and general seizures experienced during the acute and convalescent phase throughout the whole course. In addition, the children experienced various degrees of prolonged periods of fever; the clinical course was not typical of general acute encephalitis, the prognosis was poor, and the mortality and morbidity were high; therefore, it was considered to be a novel disease (2). After 15 years, in 2001, Sakuma et al (1) diagnosed and treated one case, and retrospectively analyzed 21 cases reported in the literature, and named the disease AERRPS. The following diagnostic criteria were then proposed: i) The acute phase of encephalitis lasts $>2$ weeks; ii) partial seizures accompanied with general seizures occur throughout the acute and convalescent course; iii) frequent seizures result in the 


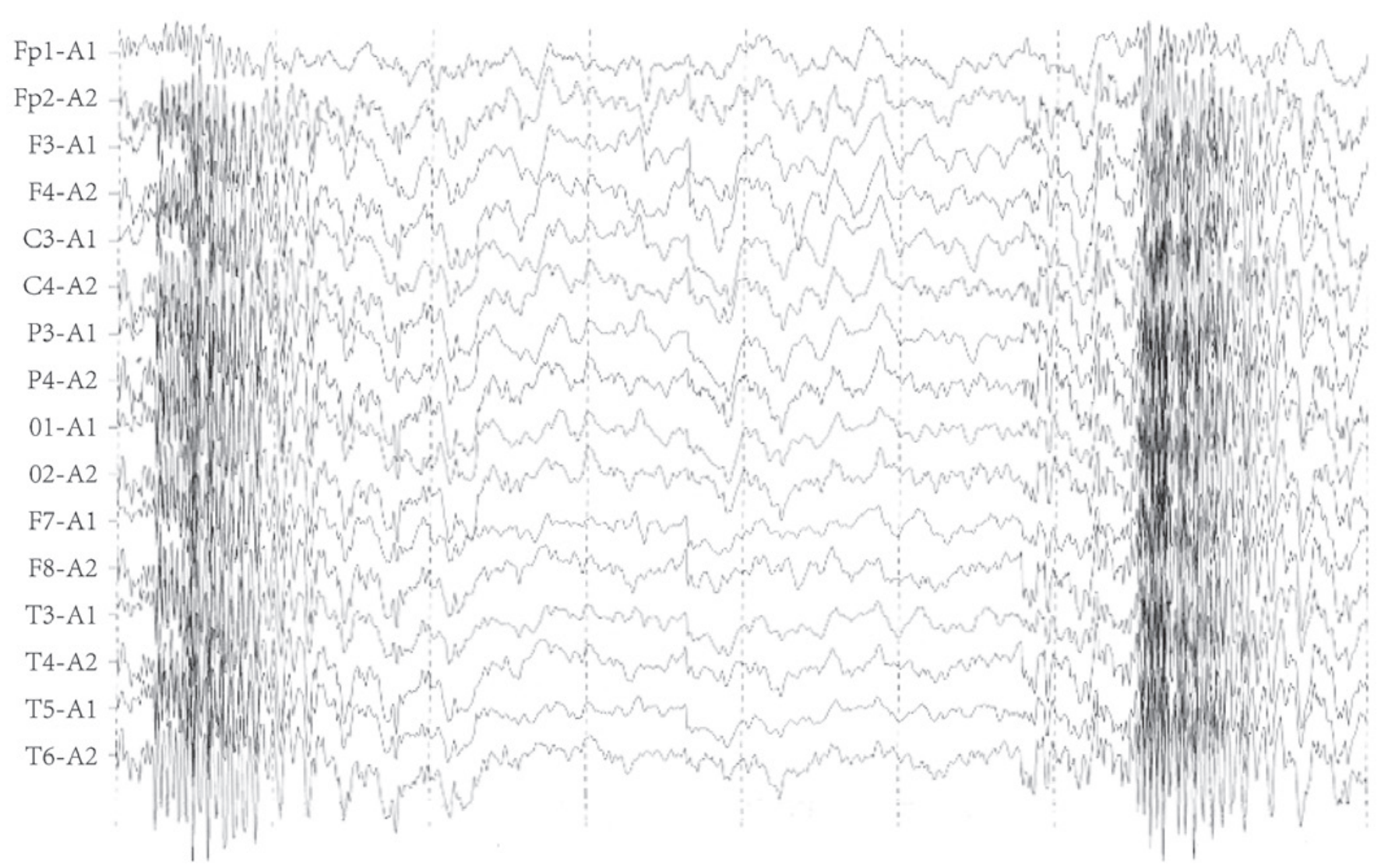

Figure 1. Combined application of muscle relaxant and anti-epileptic drugs. A large quantity of high-amplitude sharp waves and spikes are present in the electroencephalogram monitoring.

sustained state, which is more prominent in the acute phase; iv) the prominent clinical manifestation of seizures is difficult to control; v) viral encephalitis and systemic metabolic disorders are excluded. To date, the English literature has reported $>200$ AERRPS cases, including cases reported in Japan (3-12), Chinese Taiwan (13,14), Italy (15), Australia (16) and the United Arab Emirates (17); other countries have reported a small number of cases. The authors of the current study reported 4 cases in 2009 in Chinese.

To date, the cause of AERRPS remains unclear, although a previous report (2) stated that it may be caused by a viral infection in the patients' blood or CSF, no specific virus has been identified. Other previous studies $(7,10)$ reported evidence of immunological abnormalities in the patients' blood or CSF, particularly in glutamate receptor (GluR) antibodies. Wakamoto et al (10) demonstrated that the GluR, namely the $\varepsilon 2, \zeta 1$ and $\delta 2$ subtype immunoglobulin $\mathrm{G}$ antibodies, exist in the patient's blood and CSF, and that plasma interleukin (IL)-2, IL-6, IL-10, tumor necrosis factor- $\alpha$ and interferon- $\gamma$ were significantly increased. Ito et al (4) used hormone impulse and high-dose $\gamma$-globulin treatment and achieved positive results. An 11-year-old male suffered from AERRPS, and was positive for autoantibodies to the glutamate receptor Gluepsilon $2 \mathrm{IgG}$ or IgM in an examination of blood and CSF. The patient was treated with artificial respiration, thiamylal sodium, mild hypothermia therapy, steroid pulse therapy and massive gamma-globulin therapy. Afterwards, the patient had sequelae, such as postencephalitic epilepsy (the same seizures continued to recur), hyperkinesia, impairment of immediate memory, a change in character (he became sunny and obstinate), dysgraphia, and mild atrophy of the hippocampus, amygdala and cerebrum. However, the patient could still attend school. These results suggest that AERRPS may be immune-mediated autoimmune encephalitis. In the present study, the patient experienced prodromal symptoms of upper respiratory tract infections, such as fever, slight increase of elevated CSF pressure and transient increase of cell count and proteins. This suggests that AERRPS is caused by inflammatory lesions of the central nervous system; however, the symptoms were markedly different from the clinical course of general acute encephalitis. It would have been beneficial to conduct immunological tests of the CSF, but these were not performed. The clinical course experienced by the patient in the current study fully met the diagnostic criteria of AERRPS.

The neuroimaging of AERRPS exhibits no specificity $(2,18)$; the majority of patients exhibit normal or non-specific changes with regards to cranial CT or MRI in the acute phase, whereas various degrees of brain atrophy are exhibited in their recovery phase and are associated with poor prognosis. The two cranial MRIs performed on the patient in the current study showed no cerebral atrophy, results which were not concordant with previous studies. This may be related to the fact that the central nervous system in a patient of school age has not fully developed (8), thus cerebral atrophy would be more likely to cause secondary damage in children.

The most prominent feature of AERRPS that distinguishes it from general acute encephalitis is its uncontrollable epileptic seizures and poor prognosis. Almost every patient in the acute and convalescent phase of AERRPS experiences frequent 
complex partial seizures accompanied with or without secondary general seizures (8). EEG monitoring $(5,13)$ demonstrated that the majority of patients primarily exhibit slow-wave rhythm in intermittent episodes, with sustained polymorphic $\delta$ activities, and focal or fully explosive high-amplitude sharp rhythms at the onset of complex partial and secondary general seizures. Although the seizure frequency in the recovery phase is lower compared with the acute phase, one previous study (18) reported that a 6-year-old patient exhibited intermittent complex partial seizures for $>3$ years, and eventually succumbed to the seizures. The clinical features and EEG monitoring of the seizures in the patient in the present study were consistent with those of previous studies $(5,13)$. Two weeks after the onset of the seizures, despite the administration of combined various anti-epileptic drugs, including phenobarbital, phenytoin, midazolam, sodium valproate, propofol and lamotrigine, and muscle relaxants, the EEG monitoring and clinical onset showed no improvement. The anti-epileptic drugs were withdrawn after two weeks, and large doses of intravenous pentobarbital, $0.2 \mathrm{~g} / 2 \mathrm{~h}$, were administered, reaching a daily dose of $2.4 \mathrm{~g}$. When the plasma concentration of pentobarbital achieved $137 \mu \mathrm{g} / \mathrm{ml}$, the general seizures were markedly reduced and EEG monitoring exhibited the unique periodic outbreak-suppress form associated with a phenobarbital-induced coma, namely the spike-explosive suppression pattern (19). The highest blood concentration reached $198 \mu \mathrm{g} / \mathrm{ml}$, at which point the systemic and local seizures were significantly reduced to 0-2 times daily (general seizures) and 5-10 times daily (partial seizures). Following this, the dosage of phenobarbital was gradually decreased, until no general convulsive seizures occurred on day 64 of the application of large-dose phenobarbital. The patient experienced occasional partial seizures with a plasma concentration of phenobarbital maintained at $\sim 50 \mu \mathrm{g} / \mathrm{ml}$ in the convalescent phase. The intermittent episodes exhibited primarily slow-wave rhythms, accompanied by polymorphous $\delta$ and $\beta$ activities.

Since the cause of AERRPS is unknown, treatment targeting uncontrollable recurrent epileptic seizures has become the key to identifying the prognosis. Despite the option to administer combined anti-epileptic drugs and strong anesthetic, there lacks an effective means to fully control the seizures (14). It was reported that the intravenous injection of high-dose pentobarbital was effective in the majority of patients $(3,11)$, particularly in controlling general seizures in the acute phase; the maximal dose used was $>5 \mathrm{mg} / \mathrm{kg} / \mathrm{h}$, its effective blood concentration was $>40 \mu \mathrm{g} / \mathrm{ml}$ and the highest peak reached was $190 \mu \mathrm{g} / \mathrm{ml}$.

Whether AERRPS is a novel disease and the elucidation of its epidemiology and etiology remain the focus of academic controversy. Although cases of AERRPS are rare, its unique characteristics, different from general encephalitis, must be recognized clinically. In the present study, although the patient underwent long rehabilitation in his recovery phase, his intelligence, language functions and motor functions were severely impaired, and the patient ultimately succumbed to suffocation, which was consistent with the poor prognosis reported.

The reported age distribution of patients with AERRPS ranges between 5 months and 18 years; the disease primarily affects school-age children and adolescents, with one case reported of a 22 year old adult diagnosed with AERRPS (12). The patient in the present study was 46-years-old, inconsistent with the results of previous studies, suggesting that children and young adults are the primary age groups affected. However, the diagnostic criteria of AERRPS does not emphasize age as a factor; when an adult patient meets the diagnostic criteria, the possibility of AERRPS should be considered, as the early application of large-dose phenobarbital to control seizures may be the key towards improved prognosis.

\section{References}

1. Sakuma H, Fukumizu M and Kohyama J: Efficacy of anticonvulsants on acute encephalitis with refractory, repetitive partial seizures (AERRPS). No To Hattatsu 33: 385-390, 2001 (In Japanese).

2. Awaya Y and Fukuyama Y: Epilepsy sequelae of acute encephalitis: Encephalopathy (third report). Jpn J Psychiatry Neurol 40: 385-387, 1986.

3. Hamano K, Watanabe A and Kohyama J: A case of acute encephalitis with refractory, repetitive partial seizures (AERRPS) showing transient disappearance of the seizure with occurrence of choreo-ballistic movement. No To Hattatsu 35: 59-64, 2003 (In Japanese).

4. Ito H, Mori K, Toda Y, Sugimoto M, Takahashi Y and Kuroda Y: A case of acute encephalitis with refractory, repetitive partial seizures, presenting autoantibody to glutamate receptor Gluepsilon2. Brain Dev 27: 531-534, 2005

5. Saito Y, Maegaki Y, Okamoto R, Ogura K, Togawa M, Nanba Y, Inoue T, Takahashi Y and Ohno K: Acute encephalitis with refractory, repetitive partial seizures: Case reports of this unusual post-encephalitic epilepsy. Brain Dev 29: 147-156, 2007.

6. Maegaki Y, Kondo A, Okamoto R, Inoue T, Konishi K, Hayashi A, Tsuji Y, Fujii S and Ohno K: Clinical characteristics of acute encephalopathy of obscure origin: A biphasic clinical course is a common feature. Neuropediatrics 37: 269-277, 2006.

7. Okanishi T, Mori Y, Kibe T, Takahashi Y, Saito Y, Maegaki Y and Yokochi K: Refractory epilepsy accompanying acute encephalitis with multifocal cortical lesions: Possible autoimmune etiology. Brain Dev 29: 590-594, 2007.

8. Sakuma H: Acute encephalitis with refractory, repetitive partial seizures. Brain Dev 31: 510-514, 2009.

9. Sakuma H, Awaya Y, Shiomi M, Yamanouchi H, Takahashi Y, Saito Y, Sugai K and Sasaki M: Acute encephalitis with refractory, repetitive partial seizures (AERRPS): A peculiar form of childhood encephalitis. Acta Neurol Scand 121: 251-256, 2010.

10. Wakamoto H, Takahashi Y, Ebihara T, Okamoto K, Hayashi M, Ichiyama T and Ishii E: An immunologic case study of acute encephalitis with refractory, repetitive partial seizures. Brain Dev 34: 763-767, 2012.

11. Watanabe S, Okumura Y and Aiba H: A case of acute encephalitis with refractory repetitive partial seizures successfully controlled by very-high-dose phenobarbital therapy found in a boy. No To Hattatsu 46: 443-446, 2014 (In Japanese).

12. Matsuzono K, Kurata T, Deguchi S, Yamashita T, Deguchi K and Abe K: Ketogenic diet therapy is effective in encephalitis with refractory seizures. Neurol Res 36: 906-910, 2014.

13. Shyu CS, Lee HF, Chi CS and Chen CH: Acute encephalitis with refractory, repetitive partial seizures. Brain Dev 30: 356-361, 2008.

14. Lin JJ, Lin KL, Wang HS, Hsia SH and Wu CT: Effect of topiramate, in combination with lidocaine and phenobarbital, in acute encephalitis with refractory repetitive partial seizures. Brain Dev 31: 605-611, 2009

15. Specchio N, Fusco L, Claps D and Vigevano F: Epileptic encephalopathy in children possibly related to immune-mediated pathogenesis. Brain Dev 32: 51-56, 2010.

16. Dahaba AA, Liu DW and Metzler H: Bispectral index (BIS) monitoring of acute encephalitis with refractory, repetitive partial seizures (AERRPS). Minerva Anestesiol 76: 298-301, 2010.

17. Ismail FY and Kossoff EH: AERRPS, DESC, NORSE, FIRES: Multi-labeling or distinct epileptic entities? Epilepsia 52: e185-e189, 2011.

18. Fukuyama T, Inaba Y, Higuchi T, Sekiguchi Y and Ishida S: A case of sudden unexpected death in epilepsy 3 years after the onset of acute encephalitis with refractory, repetitive partial seizures. No To Hattatsu 43: 313-316, 2011 (In Japanese).

19. Hufnagel A, Burr W, Elger CE, Nadstawek J and Hefner G: Localization of the epileptic focus during methohexital induced anthesia. Epilepsia 33: 271-284, 1992. 